\title{
Hot X-ray onsets of solar flares
}

\author{
Hugh S. Hudson ${ }^{\circledR},{ }^{1,2 \star}$ Paulo J. A. Simões, ${ }^{1,3}$ Lyndsay Fletcher, ${ }^{1,4}$ Laura A. Hayes ${ }^{5}$ and Iain G. Hannah ${ }^{1}$ \\ ${ }^{1}$ SUPA School of Physics and Astronomy, University of Glasgow, Glasgow G12 8QQ, UK \\ ${ }^{2}$ Space Sciences Laboratory, UC Berkeley, 94720 CA, USA \\ ${ }^{3}$ Centro de Rádio Astronomia e Astrofísica Mackenzie, Escola de Engenharia, Universidade Presbiteriana Mackenzie, São Paulo 01302, Brazil \\ ${ }^{4}$ Rosseland Centre for Solar Physics, University of Oslo, PO Box 1029 Blindern, NO-0315 Oslo, Norway \\ ${ }^{5}$ Solar Physics Laboratory, Code 671, Heliophysics Science Division, NASA Goddard Space Flight Center, Greenbelt, MD 20771, USA
}

Accepted 2020 November 15. Received 2020 November 15; in original form 2020 July 6

\begin{abstract}
The study of the localized plasma conditions before the impulsive phase of a solar flare can help us understand the physical processes that occur leading up to the main flare energy release. Here, we present evidence of a hot X-ray 'onset' interval of enhanced isothermal plasma temperatures in the range of 10-15 MK over a period of time prior to the flare's impulsive phase. This 'hot onset' interval occurs during the initial soft X-ray increase and definitely before any detectable hard X-ray emission. The isothermal temperatures, estimated by the Geostationary Operational Environmental Satellite X-ray sensor, and confirmed with data from the Reuven Ramaty High Energy Solar Spectroscopic Imager, show no signs of gradual increase, and the "hot onset' phenomenon occurs regardless of flare classification or configuration. In a small sample of four representative flare events, we tentatively identify this early hot onset soft X-ray emission to occur within footpoint and low-lying loop regions, rather than in coronal structures, based on images from the Atmospheric Imaging Assembly. We confirm this via limb occultation of a flaring region. These hot X-ray onsets appear before there is evidence of collisional heating by non-thermal electrons, and hence challenge the standard modelling techniques.
\end{abstract}

Key words: Sun: corona-Sun: flares-Sun: X-rays.

\section{INTRODUCTION}

Often flare initiation, as seen in soft X-ray (SXR) data from the X-ray sensor (XRS) on Geostationary Operational Environmental Satellite $(G O E S)$, begins with a slow 'precursor' development phase. This can sometimes be identified with non-thermal activity (e.g. Fárník et al. 2003) or with non-thermal velocity distributions (Harra et al. 2013). The pre-flare interval is often also taken as evidence for a 'preheating' phase in which a gradual process heats a volume of flare plasma without a detectable hard X-ray (HXR) signature (e.g. Cheng et al. 1985), implying a very low flux of non-thermal electrons, if any. Systematic studies of SXR images suggested that in most cases the precursor sources could not be directly identified with the main flare (Fárník \& Savy 1998; Hudson et al. 2008), appearing near but not exactly at the flare site.

Previous conclusions about the relationship between 'precursors' and flares have dealt with varied indicators Here, we study the simple $\mathrm{X}$-ray spectral evolution, focusing on a sample of four representative events. We examine flare onset emission, where we define the term 'onset' as the pre-flare interval during which elevated GOES SXR flux is detected, but prior to the detection of any elevated HXR emission (at $>25 \mathrm{keV}$ for stronger events, and 12-25 keV for weaker ones) by the Reuven Ramaty High Energy Solar Spectroscopic Imager (RHESSI; Lin et al. 2002). Our main finding is that the GOES isothermal temperatures are significantly elevated from the very beginning of the onset phase, i.e. well before we have evidence

^E-mail: hugh.hudson@glasgow.ac.uk for collisional heating by non-thermal electrons. We cannot preclude the possibility of undetectable HXR emission, especially with a softer spectrum, in the onset time interval. We note that Awasthi \& Jain (2011) had already reported similar phenomena via the independent data set from the Solar X-Ray Spectrometer experiment (Jain et al. 2006), in a sample of 13 events.

We note that the hot onset emission precedes the impulsive-phase 'impulsive footpoint' phenomenon (McTiernan et al. 1993; Hudson et al. 1994; Mrozek \& Tomczak 2004; Fletcher et al. 2013; Graham et al. 2013; Simões, Graham \& Fletcher 2015b). This distinct feature (the footpoint SXR sources) accompanies the impulsive-phase HXR emission.

We have used extreme ultraviolet (EUV) images to search out the spatial patterns of the onset sources (Section 2.5). We have also studied the RHESSI data for the four sample events (Section 3.2), finding satisfactory qualitative agreement during the flare development, specifically in matching the isothermal-fit GOES parameters with the more complete spectroscopy possible with RHESSI.

\section{DATA}

\subsection{GOES SXR data}

The GOES series of missions has provided SXR measurements via its XRS instrument in two nominal wavelength bands (1-8 and 0.5-4 $\AA_{\text {) }}$ for many decades now. Such observations began as early as 1960 with ionization chambers onboard SOLRAD and other satellites (Dere, Horan \& Kreplin 1974; Thomas, Starr \& Crannell 1985; White, Thomas \& Schwartz 2005). The two passbands of the GOES/XRS, 
namely $1-8$ and $0.5-4 \AA$, allow for the determination of an isothermal temperature and emission measure, interpreted here in terms of the CHIANTI atomic-physics package (Dere et al. 1997) as implemented in the SOLARSOFT (Freeland \& Handy 1998) code GOES_TEM.pro. These parameters usually describe the coronal part of the flare, and specifically the plasma trapped in the system of magnetic loops made visible in SXRs by the injection of new plasma expanding upwards from the lower atmosphere due to the sudden energy release. The GOES data also have sufficient sensitivity and signal contrast to study the onset phase of a flare, often many minutes prior to the impulsive phase (Kane \& Anderson 1970). In this paper, we use these simple GOES/XRS observations to characterize the onset temperatures, at the earliest possible times permitted by the observations, and then follow up with EUV images taken by the Atmospheric Imaging Assembly (AIA; Lemen et al. 2012) onboard the Solar Dynamics Observatory (SDO; Pesnell, Thompson \& Chamberlin 2012). We also examine a flare series in which limb occultation distinguishes the coronal and chromospheric components (e.g. Hudson 1978).

Because the GOES data integrate over the whole disc (Sun-as-astar observations), all of the concurrent SXR sources will contribute to the background level for a given flare. In principle, there is no exact way to estimate this background level for such Sun-as-a-star observations, since an independent source(s) could occur at any time, in any active region that might coincidentally flare up. Bornmann (1990) had suggested a way in which to set limits on the plasma parameters with allowance for the possibility that a portion of the background (pre-flare) level could have come from the actual flaring loops themselves, and thus disappear from the background during the flare. This conservative approach, appropriate for observations unsupported by imaging, led to the TEBBS ${ }^{1}$ method (Ryan et al. 2016). Unfortunately, this algorithm inherently biases the results for the faintest flare emissions, as shown in fig. 2 of that paper. In this work, we estimate the flare background level by simply taking the local minimum of the $0.5-4 \AA$ channel immediately prior to the flare onset, the nearer the better. The actual epoch of the hot onset will depend upon flare brightness and detection threshold; for an $\mathrm{X}$-class flare occurring in low-background conditions, the GOES photometers can already detect the source at a level $0.1-1$ per cent of flare maximum flux.

We can also check the background source locations via the EUV images from SDO/AIA. Among its passbands, Fletcher et al. (2013) showed that the time series of the $131 \AA$ data provides the closest match to the GOES 1-8 ^ time series at least for the flare SOL201008-07T18:24 (M1.0). During flares, the AIA $131 \AA$ passband is dominated by the Fe XXI 128.8 $\AA$ and Fe XXIII $132.9 \AA$ lines, formed at $\log T=7.05$ and 7.15 , respectively, while the $94 \AA$ passband captures the emission from Fe XVIII 93.9 $\AA$ and Fe XX 93.8 $\AA$ formed at $\log T=6.85$ and 7.0, respectively (e.g. O'Dwyer et al. 2010).

\subsection{Event sample}

Fig. 1 shows the GOES flux, temperature, and emission measure results for four events (detailed in Table 1), chosen arbitrarily to represent fast, slow, strong, and weak flares, respectively, crudely bracketing the parameter space of rise time and flare energy. 'Fast/slow' refers to the event rise time and 'strong/weak' refers to the GOES 1-8 A peak flux values. These typical events are from the 2011 to 2014 time frame and do not represent different flare classes as such,

1 ‘Temperature and Emission measure-Based Background Subtraction’. since the parameters generally have broad, continuous distributions (e.g. Lee, Petrosian \& McTiernan 1993).

The hot onset sources appear substantially before the beginning of the impulsive phase (indicated by the dotted vertical lines in the left column panels in Fig. 1), as determined by RHESSI HXRs above $25 \mathrm{keV}$ where possible, and above $12 \mathrm{keV}$ where not. The correlation between temperature and emission measure (right column in Fig. 1) shows a roughly clockwise circulation during the main phase of the flare, ending with the cooling of the coronal loops. The hot onset emission precedes these features, appearing at the lowest emission measure but at an already elevated temperature. The cooling phase passes through the onset temperature range smoothly, indicating that the initial 10-15 MK temperature estimates are not artefactual. The data points are colour coded and mapped to the temperature curves in the middle column of Fig. 1 to indicate the time evolution of this correlation. These panels indicate that the hot onset (with temperatures around $10-15 \mathrm{MK}$ ) is associated with a low amount of plasma, with emission measure values below $10^{47} \mathrm{~cm}^{-3}$. In the flare sample discussed here, the 'fast strong' event SOL2014-0107 (bottom row of Fig. 1), for example, has a hot onset detectable more than 1 min prior to the detectable HXR emission. The GOES isothermal onset temperatures, i.e. the first valid measurements, lie well above the low-temperature range of these data (e.g. Sterling, Hudson \& Watanabe 1997; White et al. 2005). So far as the data permit us to tell, the first detected emission at these hot onset times already has a measurable temperature significantly above any observational limit.

\subsection{Uncertainties on GOES temperature measurements}

The error bars in Fig. 1 reflect both random errors, as estimated from the scatter of data at an intermediate flux level, and the digital uncertainty resulting from undersampling the true background noise, as discussed in Simões, Hudson \& Fletcher (2015a). The digital step size varies from epoch to epoch, since the different $G O E S$ satellites have different properties. For GOES-15 in the 0.5-4 Å channel, it was $6 \times 10^{-10} \mathrm{~W} \mathrm{~m}^{-2}$ equivalent, at the time of SOL2014-01-07 (peak $0.5-4 \AA$ flux $2.35 \times 10^{-5} \mathrm{~W} \mathrm{~m}^{-2}$ ). This is a crucial matter at flare onset under low-background conditions, because it often dominates the pre-flare fluctuation in the more important (in the sense of greater variance) $0.5-4 \AA$ band. Fig. 2 illustrates the noise properties for one of our illustrative events. During quiet conditions, the $0.5-4 \AA$ background levels often reflect only instrumental sources, such as thermal noise and/or unwanted radiation effects, rather than solar signal. At these times, the digital step size may exceed the intrinsic detector noise fluctuation, and this can add variance in a manner difficult to characterize in a transient. Accordingly, we have adopted the minimum digital increment as a noise term, directly added to the signal fluctuation determined via the RMS fluctuation at higher signal levels determined during a chosen interval at higher rates. Here, we take 10 data points $1 \mathrm{~min}$ after the peak of the $1-8 \AA$ channel, as indicated by the red dashed vertical lines in the left column panels of Fig. 1. We estimate the intrinsic detector noise fluctuation as the normalized standard deviation of the residuals of fitting this 10-data point interval with a fourth-order polynomial function, to which the digital noise is added linearly. These noise estimates are then added to and subtracted from the background-subtracted GOES flux (on both channels) and fed into the SOLARSOFT GOES_TEM.pro to obtain the uncertainties for the temperature and emission measure values.

Fig. 2 shows the sensitivity of the inferred hot onset temperature determination to the choice of background time interval, for the case of SOL2010-11-05. Here, the error bars just reflect the standard 
SOL2010-11-05T13:29 M1.0 (strong \& slow)
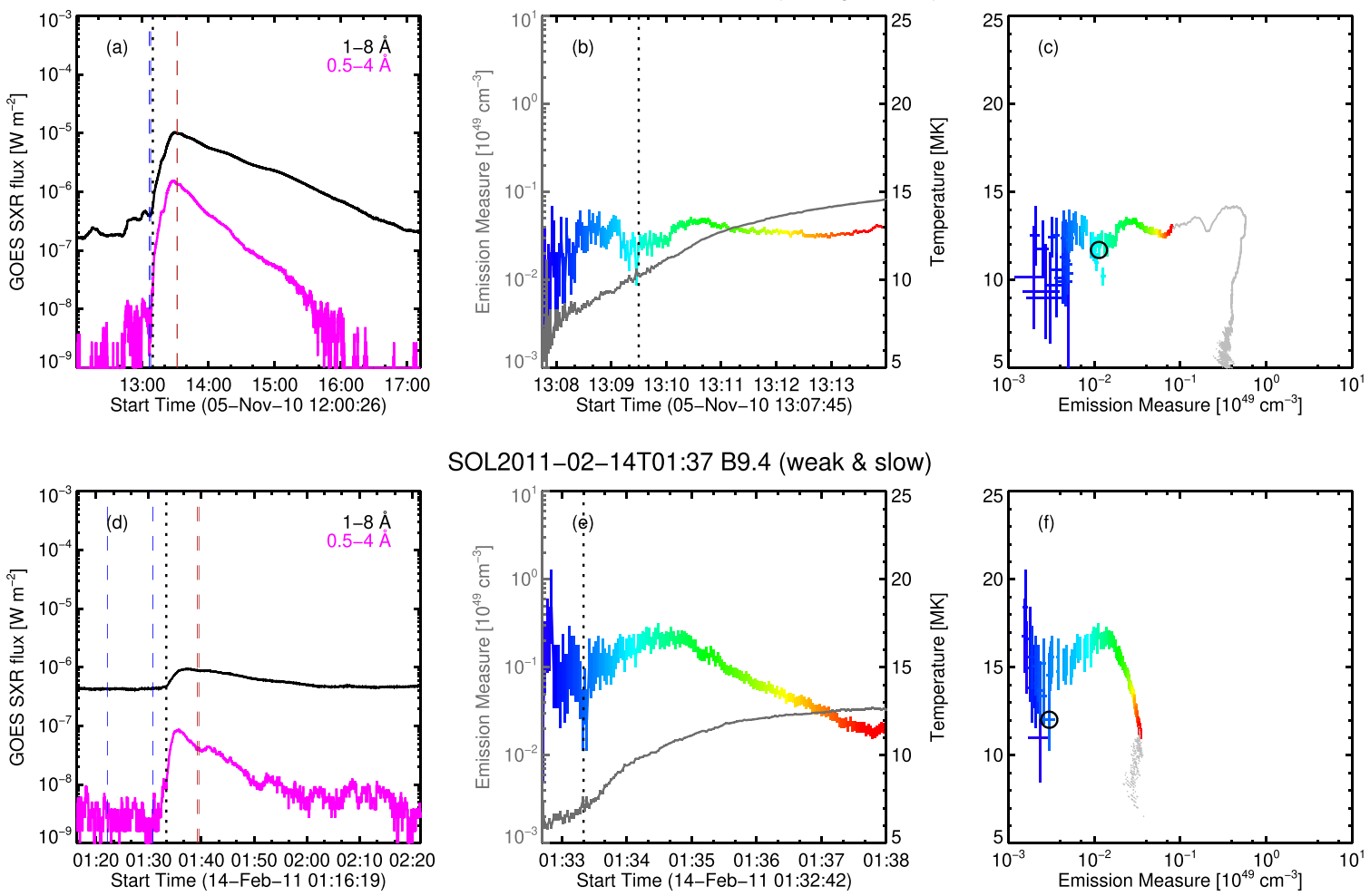

SOL2011-02-14T01:37 B9.4 (weak \& slow)
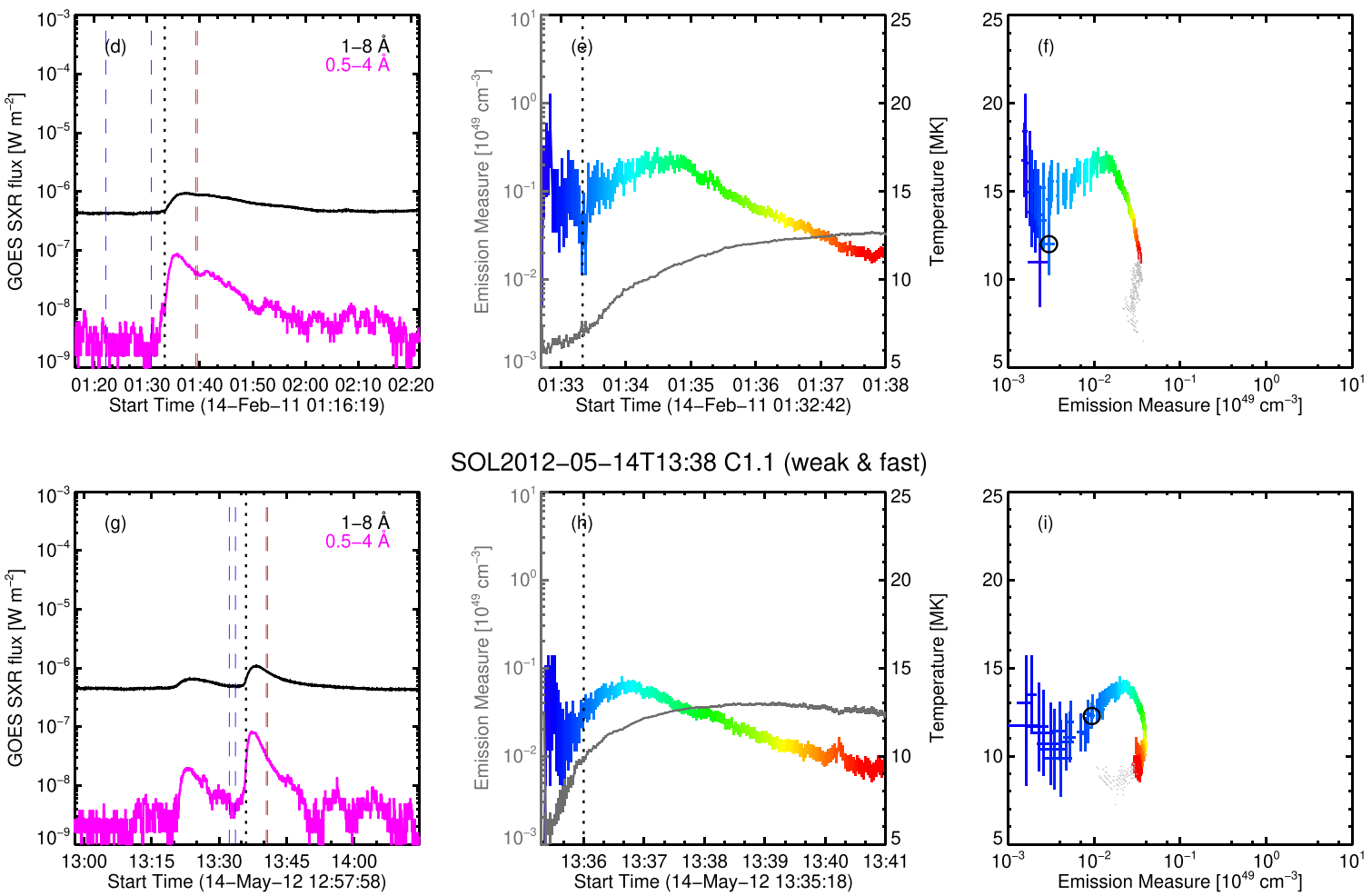

SOL2012-05-14T13:38 C1.1 (weak \& fast)
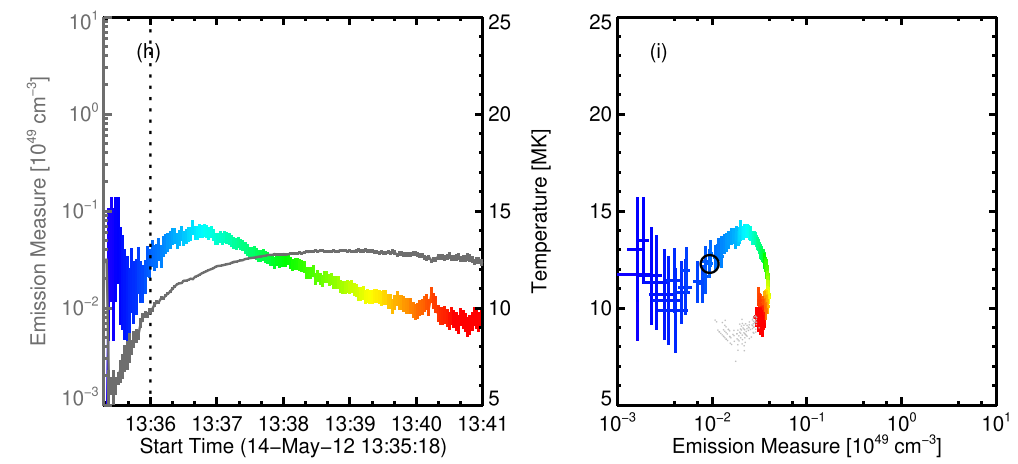

SOL2014-01-07T10:13 M7.3 (strong \& fast)
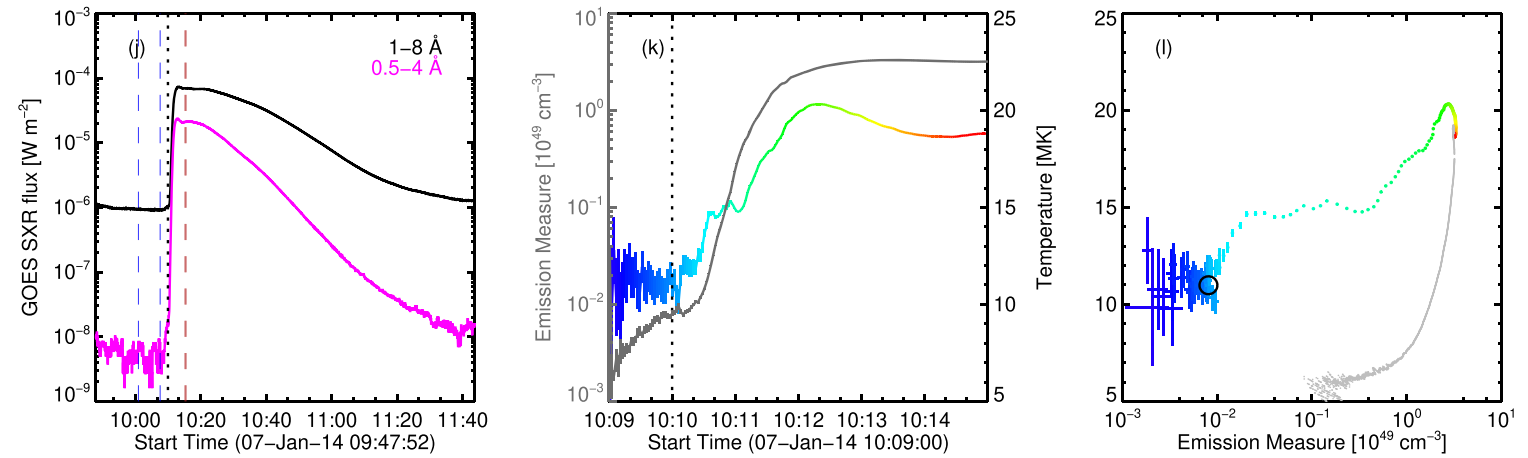

Figure 1. GOES isothermal-fit parameters for the four representative events. Left-hand column: GOES SXR flux data. The dashed lines show the time intervals for background (blue; see Table 1) and noise (red) estimation. The dotted vertical line shows the start time of the RHESSI HXR (>12 keV) emission; see Table 2. Centre column: time series of the temperature (colour coded) and the emission measure (grey). Right-hand column: correlation between temperature and emission measure. The hot onset times show up at the very beginning, at lowest emission measure and with temperatures in the $10-15 \mathrm{MK}$ range. Note that the temperature uncertainties are overestimates, as described in the text. The black circles show the parameter state at the approximate time of the dotted lines in the left-hand column plots; the colour coding matches that of the time series plot. 
Table 1. Four representative events.

\begin{tabular}{lccc}
\hline Flare (IAU) & $\begin{array}{c}\text { GOES background } \\
\text { interval (UT) }\end{array}$ & $\begin{array}{c}\text { GOES onset } \\
\text { interval (UT) }\end{array}$ & Description \\
\hline SOL2010-11-05T13:30 (M1.0) & $13: 06: 19-13: 07: 30$ & $13: 08: 00-13: 09: 30$ & Strong, slow \\
SOL2011-02-14T01:37 (B9.4) & $01: 22: 09-01: 30: 47$ & $01: 32: 00-01: 33: 20$ & Weak, slow \\
SOL2012-05-14T13:38 (C1.1) & $13: 32: 16-13: 33: 38$ & $13: 35: 25-13: 36: 00$ & Weak, fast \\
SOL2014-01-07T10:13 (M7.3) & $10: 00: 57-10: 07: 36$ & $10: 09: 05-10: 10: 00$ & Strong, fast \\
\hline
\end{tabular}
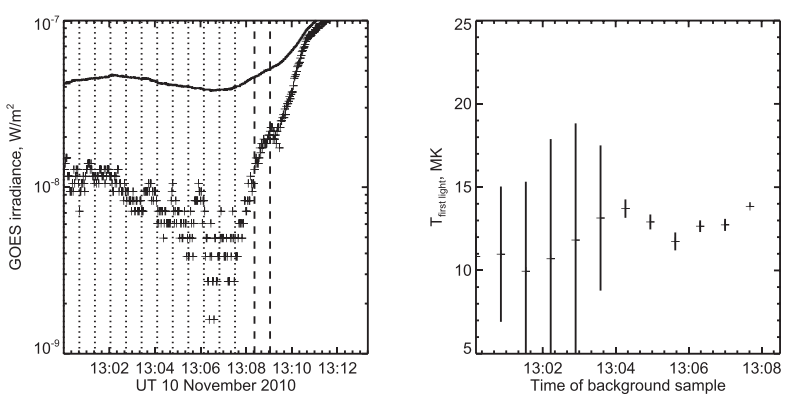

Figure 2. Illustration of the sensitivity of the hot onset temperature, at a fixed time interval, to different choices of background interval in the analysis of the GOES data for SOL2010-11-05. Left: the two GOES time series (1-8 $\AA$ divided by 10) with dotted lines showing different choices of background interval. Note the dominance of digitization error at low flux levels in the 0.5-4 A channel; right: the inferred temperature for the interval between the two dashed lines, plotted against the time at which each 20 -s background interval was taken.

deviation of the 20-point sample at a fixed time interval, as shown by the dashed lines. The large error bars here show the observed standard deviations; the earliest background samples result in some non-physical flux ratios, for which GOES_TEM.pro returns a default $4 \mathrm{MK}$, and are thus data artefacts. Generally, the closer in time to the measurement, the more trustworthy the background information should become.

\subsection{Time histories}

The two parameters derived from the isothermal GOES fits, temperature and emission measure, allow us to trace out an event's time evolution in a correlation plot of the temperature and emission measure parameters, a technique often used to illustrate the different flare phases, as shown in the right-hand panels of Fig. 1. The hot onset interval clearly stands out in each case, with little variation of temperature as the emission measure slowly grows. For SOL201401-07 (M7.8), the emission measure of the hot onset source does not exceed 1 per cent of its ultimate maximum. As expected, the start of the impulsive phase corresponds to a large increase in emission measure simultaneously with the presence of higher temperatures, in the pattern expected from the Neupert effect (Neupert et al. 1967); see, for example, fig. 7 of Raftery et al. (2009).

The right column panels in Fig. 1 show only the beginning of each event with a colour-coded time for clarity. We also note that the completion of the trajectory in grey shows that the cooling branch of the diagram has no peculiarities in the temperature range of the hot onset. The late phase, densely sampled because of its relatively slow evolution, shows the pattern expected of 'overdense' flare loops cooling as a result of the cessation of major energy release (Serio et al. 1991; Cargill, Mariska \& Antiochos 1995; Raftery et al. 2009).

\subsection{EUV/UV images}

The GOES time series (Fig. 1) identify the time range of the hot onset interval for each of the four representative flares, and we track these spatially via the corresponding AIA 94 or $131 \AA$ images in Fig. 3. In each panel, we show a difference image between the onset time and the background time and then overlay with contours from the closest AIA $1700 \AA$ Amages to the onset time, highlighting the chromospheric flare emission (Simões et al. 2019). The close association of the bright and localized sources from a high-temperature plasma (94 or $131 \AA$ ) and chromospheric footpoints (1700 ̊ contours) suggests the presence of plasma of temperatures around $10 \mathrm{MK}$ at the flare footpoints even at these early stages of flares. As expected, the images of the two 'fast' events (upper left, lower right) show that they are smaller physically. The AIA images were processed using the standard software (Boerner et al. 2012).

These images confirm that the onset source regions include footpoints, identifiable at this resolution with subsequent flare footpoint sources. Some loop emission also appears. One of the three cases shows a simple double footpoint pattern, but the others have multiple regions appearing simultaneously in these single snapshot images at exposure times of $2.9 \mathrm{~s}$.

Figs 4 and 5 examine the time variations of image features for SOL2011-02-14, in order to quantify the EUV fluxes in the footpoints relative to the main body of the flare. Note the presence of multiple compact sources in this event, in the hot onset interval. These identify well with the footpoints of faint loop features, but do not show an orderly ribbon organization. The clear identification of the footpoints in both spectral bands suggests broad contributions from multithermal plasmas (e.g. Simões et al. 2015b). The EUV $131 \AA$ and UV $1700 \AA$ intensity of each identified footpoint, indicated by the coloured boxes, is summed to construct the flux time series. In Fig. 4, the pink and purple boxes mark regions of the coronal loops connecting the footpoints; their $131 \AA$ fluxes show the typical gradual rise originated from the hot plasma filling these loops, which display no emission at UV $1700 \AA$. On the other hand, the footpoints (marked by the rest of the coloured boxes) do show the emission rising in both 131 and $1700 \AA$ as early as 01:32:00 UT, with both having a more impulsive development, reaching their maximum values after 01:34 UT, very close to the maximum of the impulsive phase of this event as indicated by the peak of the HXR emission.

These comparisons are consistent with the conclusion that 10 $15 \mathrm{MK}$ temperatures appear in the footpoint sources simultaneously with the 'typical' chromospheric temperatures.

Quantitative analysis of the source fluxes in terms of their physical parameters lies outside the scope of this work, but we can readily compute the ratios of emission from footpoint and loop regions in the two bands, as shown in Fig. 5. In this figure, $F_{\text {total }}$ represents the flux of the entire region's field of view (Fig. $4 \mathrm{a}$ ), $F_{\mathrm{FP}}$ is the total flux from all the footpoint boxes in Fig. 4(a), and $F_{\text {total }}-$ $F_{\mathrm{FP}}$ gives an estimate of the emission originating only from the loops. The ratio $F_{\mathrm{FP}} / F_{\text {total }}$ (in grey) confirms the relatively strong 
(a) SOL2010-11-05 (strong \& slow)

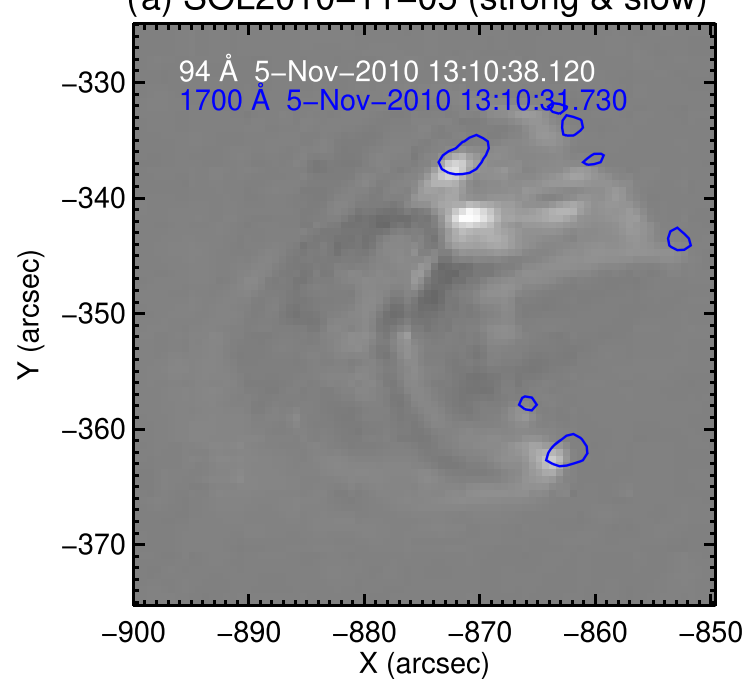

(c) SOL2012-05-14 (weak \& fast)

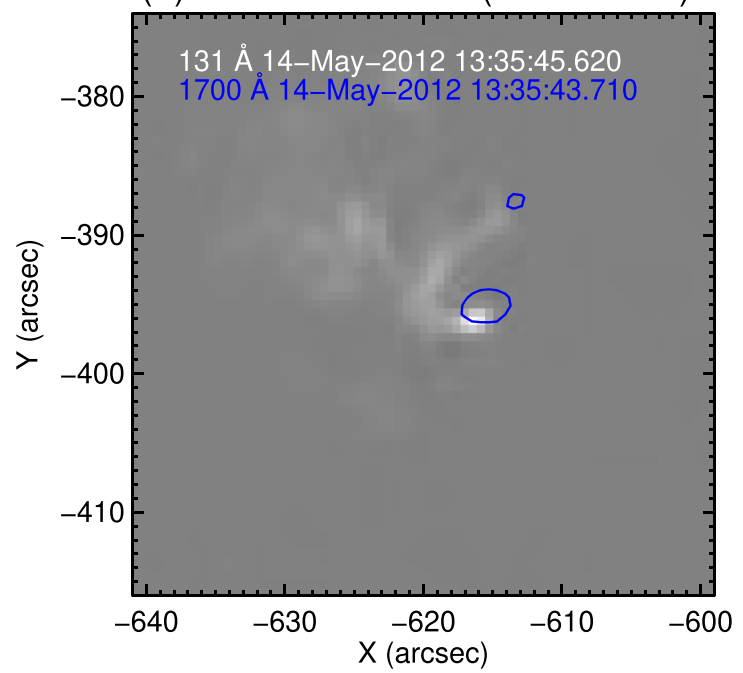

(b) SOL2011-02-14 (weak \& slow)

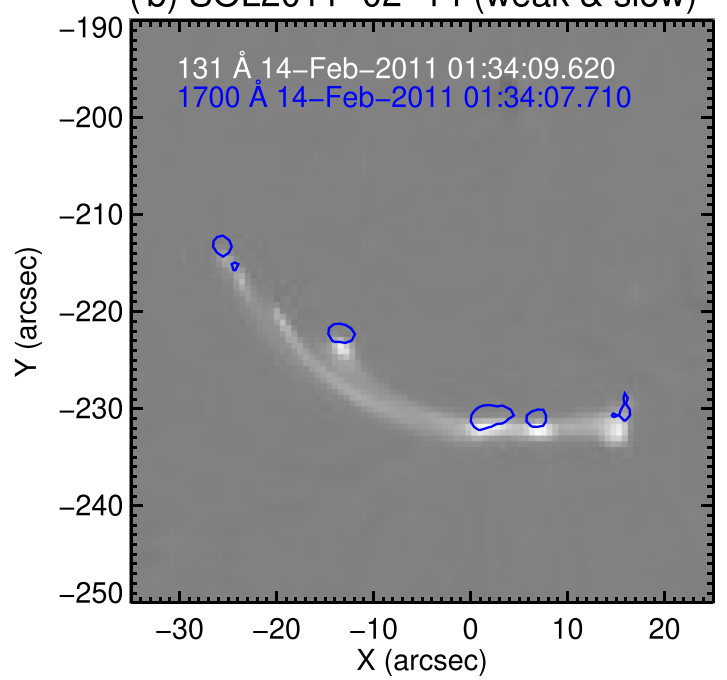

(d) SOL2014-01-07 (strong \& fast)

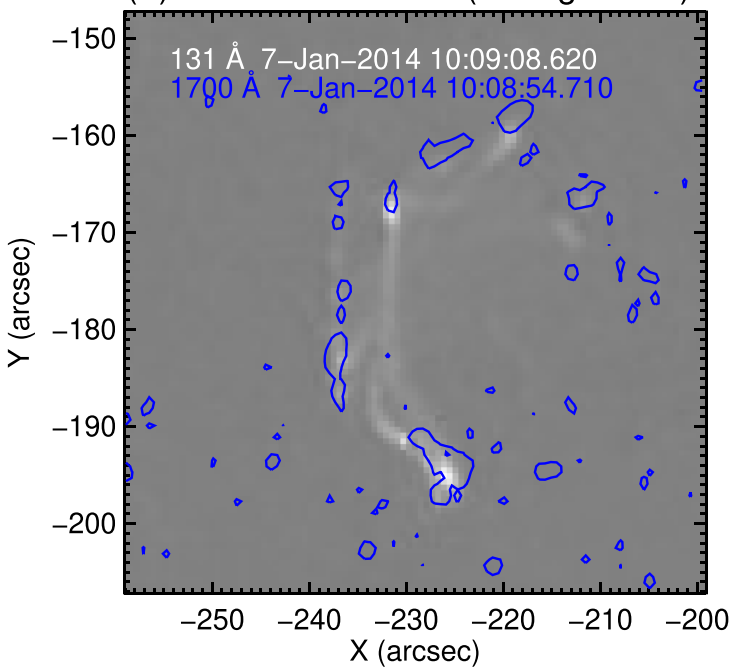

Figure 3. AIA difference images for a hot channel (94 or $131 \AA$ ), overlaid with $1700 \AA$ contours at 20 per cent of the image maximum taken from a running-difference image. (a) SOL2010-11-05, base image at 13:04:02 UT. (b) SOL2011-02-14, base image at 01:28:09 UT. (c) SOL2012-04-14, base image at 13:33:09 UT. (d) SOL2014-01-07, base image at 10:06:20 UT.

contributions (at the 20-50 per cent level) from the footpoint regions in the hot onset interval. Future studies of the hot onset phenomenon should make use of the Del Zanna (2013) techniques for isolating specific emission lines from AIA multiband data, for example, and furthermore use Hinode/XRT observations to clarify the temperature structures associated with the hot onset sources. Because of their novelty, we note that the emission-measure distributions may differ from those of ordinary flare footpoints and loop structures.

\section{CONFIRMATION OF THE HOT ONSET PROPERTY}

\subsection{Occulted flares}

The image analysis in Section 2.5 shows that we can identify the hot onset X-ray sources with corresponding AIA $1700 \AA$ features, consistent with chromospheric heights rather than a coronal origin. For a flare with footpoints occulted by the limb, just prior to or just after a limb passage, we would expect different properties. The footpoint regions in such occulted flares cannot be seen and the SXR emission must therefore come from large-scale loops (the flare arcade). In major events, these tend to show temperatures higher than the 10-15 MK hot onset range (Garcia 1994). We confirm this in Fig. 6, which shows how the onset temperature varies as National Oceanic and Atmospheric Administration (NOAA) active region 11748 transited the east limb in 2013 May. The coronal sources seen before limb transit (approximately mid-day 2013 May) have temperatures in the 15-25 MK range, depending on flare magnitude, while the on-disc sources seen after the transit tend to have temperatures in the $10-15 \mathrm{MK}$ range. This sample also helps us to confirm the universality of the hot onset phenomenon since it consists of additional events selected without selection bias. 
AIA 131 diff. SOL2011-02-14 (B9.4)
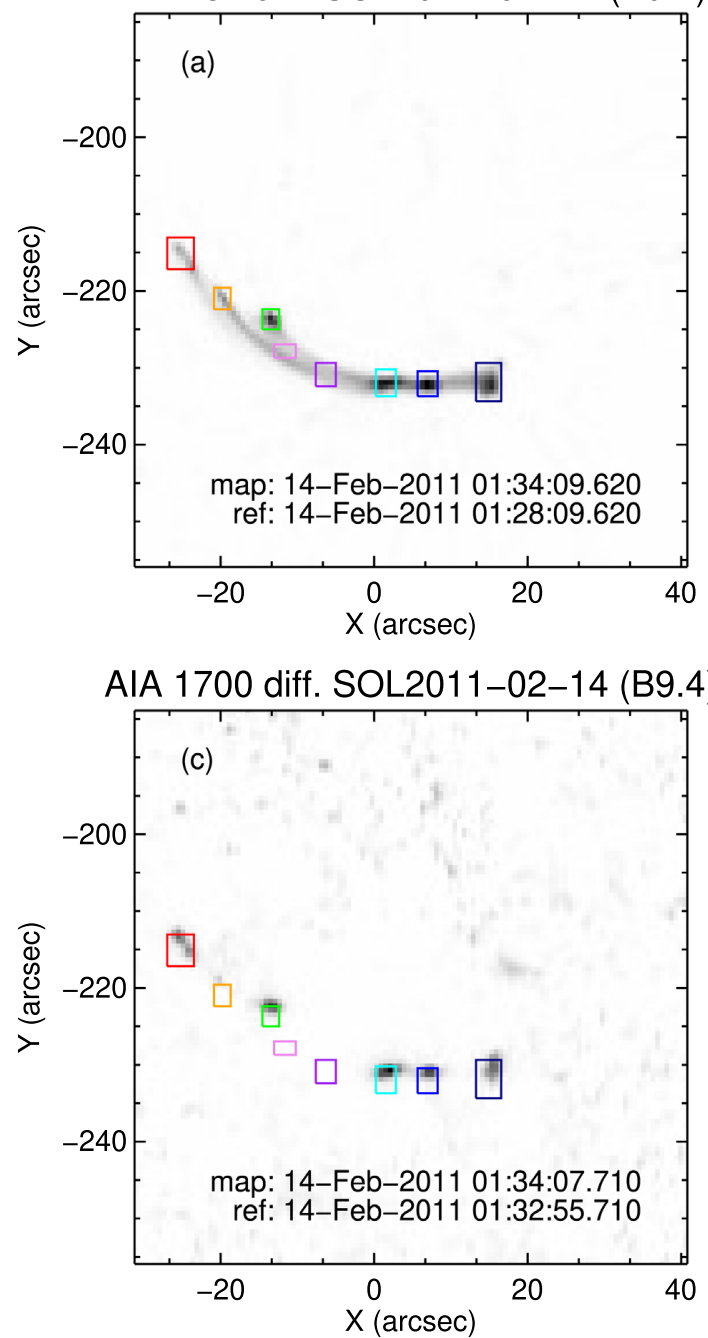
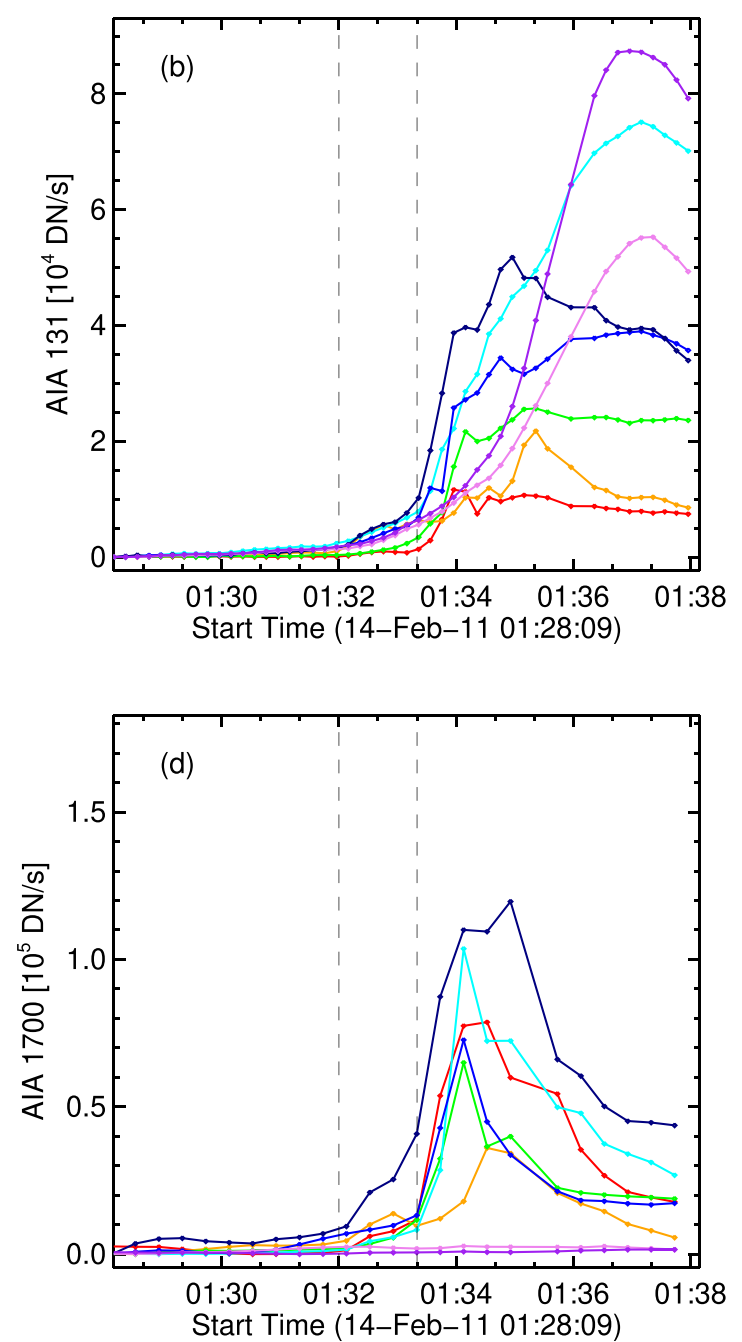

Figure 4. Identification of footpoints from AIA $131 \AA$ (top) and $1700 \AA$ images (bottom), with images on the left and their relative flux contributions on the right. The dashed vertical lines show the onset interval, identified from the GOES SXR data (see Table 1).

\subsection{RHESSI observations}

The RHESSI X-ray data provide a check on the temperatures obtained from GOES. We have analysed these observations for the four sample events, as shown in Fig. 7, where we show the light curves in $3-6,6-12$, and $12-25 \mathrm{keV}$ for each event along with a fitted spectrum. The light curve for the highest energy band (12-25 keV) typically has a non-thermal interpretation, with the lower energy bands reflecting predominantly thermal emission. The light curves can be shown in full for the SOL2011-02-14 and SOL2-12-05-14 flares, but for the larger flares (SOL2010-11-05 and SOL2014-01-07) we only show the initial period before RHESSI's attenuating shutter comes in (after the start of HXR emission in both cases). In all events, there is a steady increase in the thermal light curves during the GOES hot onset time, and it is over this time that we fit an RHESSI X-ray spectrum. In Fig. 7, we show the background spectrum from a pre-onset time, and the onset spectrum with this background subtracted. The spectral fit uses the F_VTH.PRO thermal model in the Object Spectral Executive (OSPEX software; Schwartz et al. 2002). The resulting fit parameters and uncertainties are given in Fig. 7 and Table 2. In each case, the RHESSI spectrum shows a temperature around 12-15 MK, consistent with the GOES temperatures derived for these flares. Added to this thermal model is the DRM_MOD.PRO 'pseudo-function' that accounts for the degraded detector performance (increased noise and poorer energy resolution due to radiation damage) that is present during these flares. The figure legends show the non-defaulted parameter set for this function for each case. This also requires the analysis to be performed for single RHESSI detectors; the data shown in Fig. 7 are for one of the better-performing detectors during each flare, which is Detector 9 for the first three events, but Detector 1 during the last event. Several of the other detectors produce similar but noisier results. In some of the flare spectra shown in Fig. 7, there is a slight excess in counts over the model $>10 \mathrm{keV}$, which might be indicative of very weak HXR emission, but in each case this signal is less than $2 \sigma$ above the background. The strongest case of this is for SOL2014-01-07, but RHESSI degradation was relatively severe at the time of this event. This degradation is also the likely reason for the poorer consistency between GOES and RHESSI temperatures in this event compared to the other flares. 

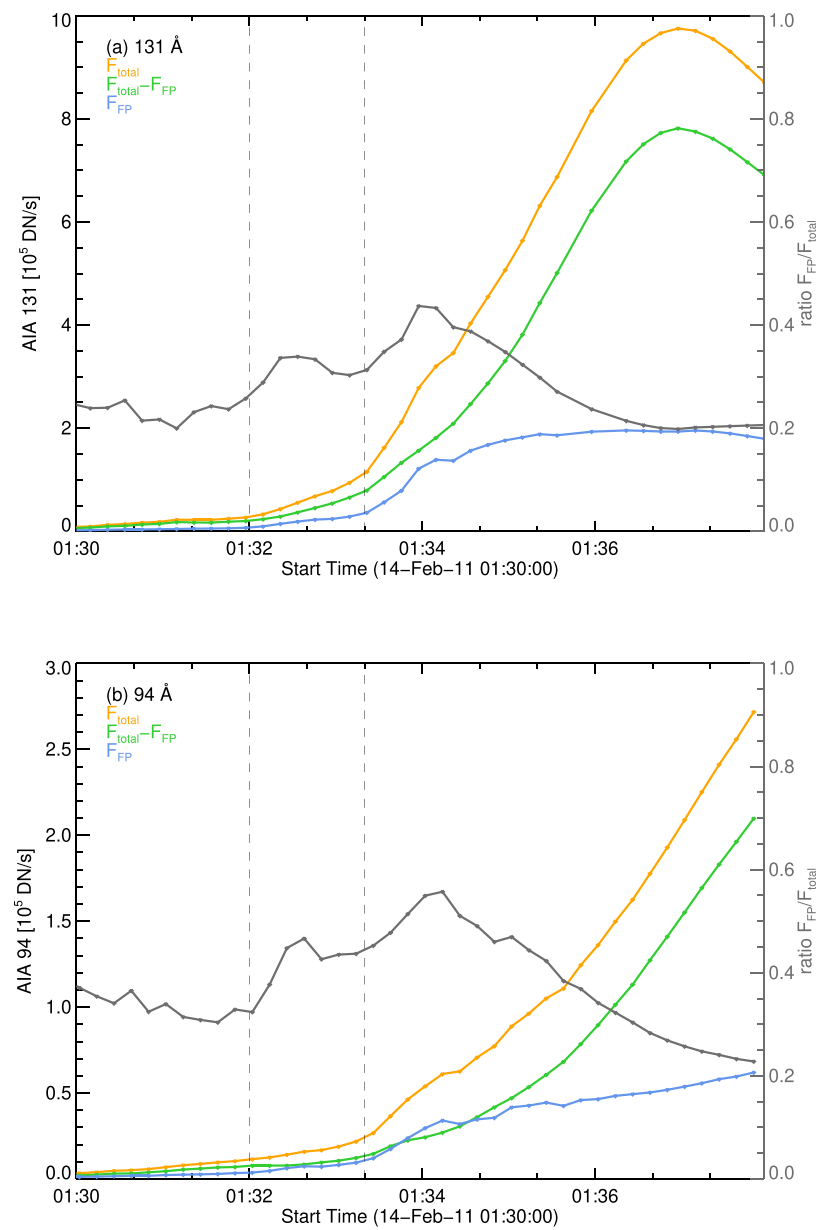

Figure 5. AIA 131 and $94 \AA$ total flux (yellow), flux in the boxed footpoint source regions defined in Fig. 4 (blue), and the difference between them as an estimate of the emission from the coronal loops (green). The ratio of footpoint flux to total flux is shown in black. The dashed vertical lines show the onset interval, identified from the GOES SXR data (see Table 1).

\subsection{Summary}

We summarize the observations described above briefly as follows:

(i) The SXR onsets of solar flares detected by GOES/XRS tend to have elevated temperatures already at the time of initial detectability.

(ii) The temperatures of these sources range from 10 to $15 \mathrm{MK}$ and do not depend strongly on flare magnitude or configuration.

(iii) These 'hot onset' sources may form in the lower atmosphere, including multiple simultaneous footpoint brightenings that appear prior to the impulsive phase and make a substantial contribution to the overall high-temperature signature.

(iv) The onset high temperatures appear within $10 \mathrm{~s}$ of the flare's detectable onset in the GOES $0.5-4 \AA$ channel, and vary only slowly over the hot onset interval, for a duration of tens of seconds to minutes.

(v) We have confirmed the GOES results with the RHESSI data.

\section{DISCUSSION}

What physical conditions prevail in the hot onset sources? A systematic study is beyond the scope here, but we can comment on

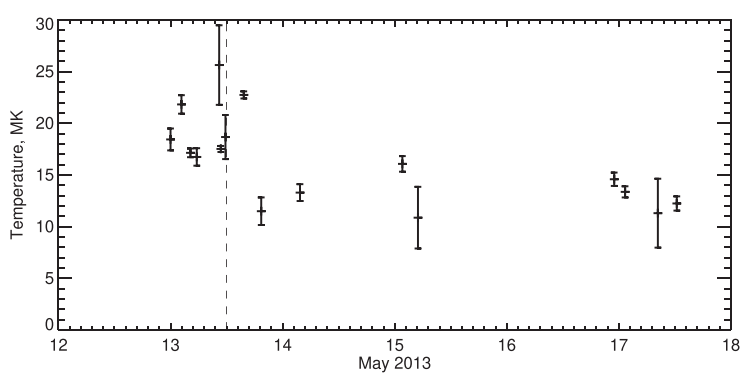

Figure 6. Earliest GOES temperatures of flares measured during the east limb transit of NOAA AR 11748 in 2013, with the approximate time (mid-day May 13) of the transit indicated by a vertical dashed line. The higher temperatures for the occulted flares are consistent with the higher temperatures of flare arcades on larger spatial scales, whereas the 10-15 MK onset sources have been occulted. Note that this plot omits SOL2013-05-14 (X3.2) because of background confusion.

the densities obtained by comparing the emission measures (Fig. 1) with the image scales seen in Fig. 3. A typical emission measure for a hot onset footpoint source is about 20 to 40 per cent (Fig. 5) of $n_{\mathrm{e}} n_{\mathrm{i}} V=10^{47} \mathrm{~cm}^{-3}$ (Fig. 1), where $n_{\mathrm{e}}$ and $n_{\mathrm{i}}$ are the electron and ion densities, respectively. Assuming $n_{\mathrm{e}}=n_{\mathrm{i}}$ and volume $V=10^{8}$ $\times 10^{8} \times 10^{7} \mathrm{~cm}^{3}$ (based on typical sizes of the hot footpoints), one finds $(3.0<n<4.7) \times 10^{11} \mathrm{~cm}^{3}$, consistent with a chromospheric region. The SOLARSOFT temperature estimates, for both the GOES and RHESSI data, assume collisional ionization equilibrium (e.g. Bradshaw 2009). This may be a reasonable assumption for these hot, dense chromospheric sources, in the absence of evidence for a significant role for collisional excitation by non-thermal electrons. However, a full exploration with a dynamic model capable of tracking the ionization states in detail should follow; in particular, the lineto-continuum ratio may differ considerably in regions of partial ionization.

\section{CONCLUSIONS}

Our work with the GOES SXR observations in the earliest detectable stages of solar flares has revealed an unexpectedly common temperature behaviour, with flares of all magnitudes starting at temperatures of 10-15 MK with no sign of gradual temperature increase. We have found this behaviour in a set of four representative events with diverse properties, plus ten further examples in the de-occulting active region AR 11748. This sample establishes that this pattern is the rule, rather than the exception, but we would like to see a broader study carried out. We suggest that these early hot sources are mainly footpoints and low-lying loops that subsequently become parts of the structure of the flare.

The behaviour of these sources may explain some of the features of the frequently reported flare precursors as seen in other ways. They offer a substantial challenge (and an opportunity) for standard modelling techniques. The recognition of the hot onset phenomenon immediately challenges the modelling as carried out with 1D radiation hydrodynamics in the standard framework (Nagai 1980, et seq.). So far as we are aware, this modelling approach has not actually predicted the behaviour that we observe. In this study, we have not made a quantitative estimate of the effective heating time, such as the quantity $[\mathrm{d}(\ln (T)) / \mathrm{d} t]^{-1}$, but note that this would provide a benchmark property for numerical simulations. We suggest that identifying the physical parameters responsible for the 

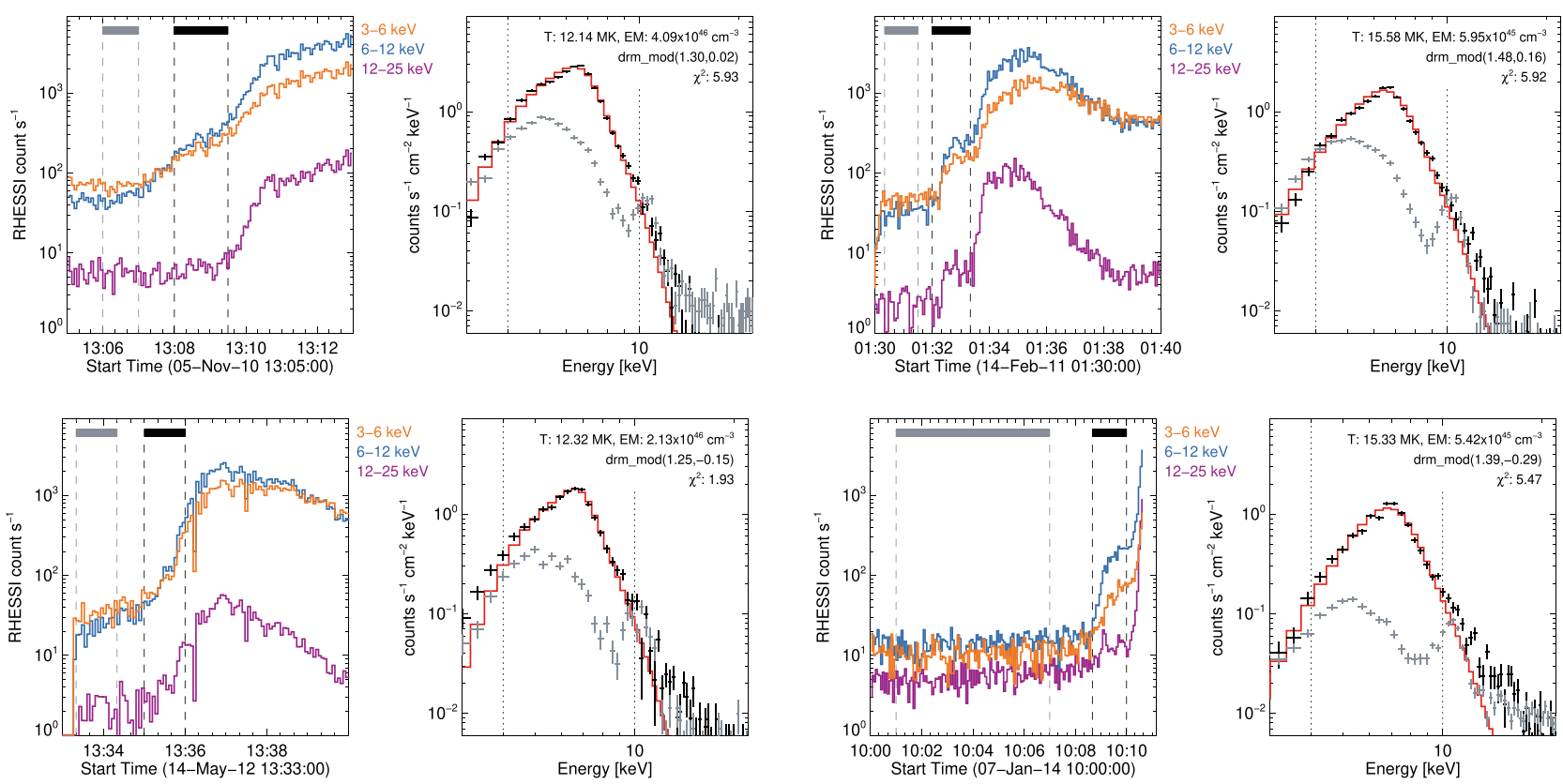

Figure 7. RHESSI light curves and fitted spectra for each of the four flares. The light curves show the RHESSI emission in three different energy bands, and indicate the time range used for the background (grey dashed vertical lines and bar) and onset (black dashed vertical lines and bar) spectra. The spectra for the background time (grey data points) and background subtracted data (black points) are shown and fitted with an isothermal model (red, parameters given) over 4-10 keV (blacked dotted vertical line). The legends for each figure give the resulting thermal fit parameters, the non-defaulted parameters used for DRM_MOD.PRO, and the resulting $\chi^{2}$ value.

Table 2. GOES/RHESSI temperature comparisons.

\begin{tabular}{lcccc}
\hline Flare (IAU) & $\begin{array}{c}\text { RHESSI background } \\
\text { interval (UT) }\end{array}$ & $\begin{array}{c}\text { RHESSI onset } \\
\text { interval (UT) }\end{array}$ & \multicolumn{2}{c}{ Temperature (MK) } \\
& GOES & RHESSI \\
\hline SOL2010-11-05T13:30 & $13: 06: 00-13: 07: 00$ & $13: 08: 00-13: 09: 30$ & $12.2 \pm 1.1$ & $12.14 \pm 0.52$ \\
SOL2011-02-14T01:37 & $01: 30: 20-01: 31: 30$ & $01: 32: 00-01: 33: 20$ & $14.5 \pm 1.7$ & $15.58 \pm 1.15$ \\
SOL2012-05-14T13:38 & $13: 33: 20-13: 34: 20$ & $13: 35: 00-13: 36: 00$ & $11.6 \pm 0.9$ & $12.32 \pm 0.49$ \\
SOL2014-01-07T10:13 & $10: 01: 00-10: 07: 00$ & $10: 08: 40-10: 10: 00$ & $11.1 \pm 0.6$ & $15.33 \pm 1.85$ \\
\hline
\end{tabular}

observed pattern should be a primary task for future work with these models, including consideration of Alfvénic energy transport (Fletcher \& Hudson 2008). The hot onset sources have diagnostic importance for the models simply because they occur first, and thus avoid confusion with the subsequent development of coronal flare loops. We emphasize that the hot onset emission precedes the impulsive-phase 'hot footpoint' phenomenon and differs from it (McTiernan et al. 1993; Hudson et al. 1994; Mrozek \& Tomczak 2004; Fletcher et al. 2013; Graham et al. 2013; Simões et al. 2015b). We do not think that the common and possibly universal nature of this phenomenon has been previously reported, but remark that the signatures certainly have appeared in individual events reported earlier, even if not noted as having any generality, for example by Cheng et al. (1985) and Falewicz, Siarkowski \& Rudawy (2011). The latter indeed drew the conclusion that a standard electron-beam model could fully explain all of the observed parameter evolution if the low-energy cut-off parameter is allowed to vary freely, but our findings disagree with that. Because of the absence of observable HXR emission, this phenomenon suggests the existence of a flare heating process that is physically different from that of the impulsive phase. We have confirmed that RHESSI observations also show these early high temperatures and look forward to a more detailed analysis of the HXR properties, either with RHESSI or with more sensitive focusing optics, such as those pioneered by Focusing Optics X-ray Solar Imager (Krucker et al. 2009) and NuSTAR (Grefenstette et al. 2016), or especially with future missions such as Fundamentals of Impulsive Energy Release in the Corona Explorer (Shih et al. 2019).

\section{ACKNOWLEDGEMENTS}

We thank NOAA's Janet Machol and Rodney Viereck, and NASA's Richard Schwartz and Kim Tolbert, for maintaining the GOE. S/XRS archive in such a scientifically useful format via SOLARSOFT. PJAS acknowledges support from the Fundo de Pesquisa Mackenzie (MackPesquisa), CNPq (contract 307612/2019-8), and FAPESP, the Sao Paulo Research Foundation (contract 2013/24155-3). IGH is supported by a Royal Society University Fellowship. LF acknowledges support from UK Research and Innovation's Science and Technology Facilities Council under grant award numbers ST/P000533/1 and ST/T000422/1. LAH is supported by an appointment to the NASA Postdoctoral Program at Goddard Space Flight Center, administered by USRA (the Universities Space Research Association) through a contract with NASA. HSH expresses thanks to the University of Glasgow for hospitality. 


\section{DATA AVAILABILITY}

All data used in this paper reside in the public domain: for example, for GOES, access via https://www.ngdc.noaa.gov/stp/satellite/goes /index.html; for RHESSI, via https://hesperia.gsfc.nasa.gov/ssw/hes si/doc/guides/hessi_data_access.htm; and for SDO/AIA, via https: //sdo.gsfc.nasa.gov/data/.

\section{REFERENCES}

Awasthi A. K., Jain R., 2011, in Astron. Soc. India Conf. Ser, 2, 297

Boerner P. et al., 2012, Sol. Phys., 275, 41

Bornmann P. L., 1990, ApJ, 356, 733

Bradshaw S. J., 2009, A\&A, 502, 409

Cargill P. J., Mariska J. T., Antiochos S. K., 1995, ApJ, 439, 1034

Cheng C. C., Pallavicini R., Acton L. W., Tandberg-Hanssen E., 1985, ApJ, 298, 887

Del Zanna G., 2013, A\&A, 558, A73

Dere K. P., Horan D. M., Kreplin R. W., 1974, J. Atmos. Terr. Phys., 36, 989 Dere K. P., Landi E., Mason H. E., Monsignori Fossi B. C., Young P. R., 1997, A\&AS, 125, 149

Falewicz R., Siarkowski M., Rudawy P., 2011, ApJ, 733, 37

Fárník F., Savy S. K., 1998, Sol. Phys., 183, 339

Fárník F., Hudson H. S., Karlický M., Kosugi T., 2003, A\&A, 399, 1159

Fletcher L., Hudson H. S., 2008, ApJ, 675, 1645

Fletcher L., Hannah I. G., Hudson H. S., Innes D. E., 2013, ApJ, 771, 104

Freeland S. L., Handy B. N., 1998, Sol. Phys., 182, 497

Garcia H. A., 1994, Sol. Phys., 154, 275

Graham D. R., Hannah I. G., Fletcher L., Milligan R. O., 2013, ApJ, 767, 83

Grefenstette B. W. et al., 2016, ApJ, 826, 20

Harra L. K., Matthews S., Culhane J. L., Cheung M. C. M., Kontar E. P., Hara H., 2013, ApJ, 774, 122

Hudson H. S., 1978, ApJ, 224, 235

Hudson H. S., Strong K. T., Dennis B. R., Zarro D., Inda M., Kosugi T., Sakao T., 1994, ApJ, 422, L25

Hudson H. S., Hannah I. G., Deluca E. E., Weber M., 2008, in Sarah A. M., John M. D., Louise K. H., eds, ASP Conf. Ser. Vol. 397, Physical
Conditions in Coronal Structures About to Flare. Astron. Soc. Pac., San Francisco, p. 130

Jain R., Joshi V., Kayasth S. L., Dave H., Deshpande M. R., 2006, JA\&A, 27, 175

Kane S. R., Anderson K. A., 1970, ApJ, 162, 1003

Krucker S. et al., 2009, in O’Dell S. L., Pareschi G., eds, Proc. SPIE Conf. Ser. Vol. 7437, Optics for EUV, X-Ray, and Gamma-Ray Astronomy IV. SPIE, Bellingham, p. 743705

Lee T. T., Petrosian V., McTiernan J. M., 1993, ApJ, 412, 401

Lemen J. R. et al., 2012, Sol. Phys., 275, 17

Lin R. P. et al., 2002, Sol. Phys., 210, 3

McTiernan J. M., Kane S. R., Loran J. M., Lemen J. R., Acton L. W., Hara H., Tsuneta S., Kosugi T., 1993, ApJ, 416, L91

Mrozek T., Tomczak M., 2004, A\&A, 415, 377

Nagai F., 1980, Sol. Phys., 68, 351

Neupert W. M., Gates W., Swartz M., Young R., 1967, ApJ, 149, L79

O’Dwyer B., Del Zanna G., Mason H. E., Weber M. A., Tripathi D., 2010, A\&A, 521, A21

Pesnell W. D., Thompson B. J., Chamberlin P. C., 2012, Sol. Phys., 275, 3

Raftery C. L., Gallagher P. T., Milligan R. O., Klimchuk J. A., 2009, A\&A, 494, 1127

Ryan D. F., Dominique M., Seaton D., Stegen K., White A., 2016, A\&A, 592, A133

Schwartz R. A., Csillaghy A., Tolbert A. K., Hurford G. J., McTiernan J., Zarro D., 2002, Sol. Phys., 210, 165

Serio S., Reale F., Jakimiec J., Sylwester B., Sylwester J., 1991, A\&A, 241, 197

Shih A. Y. et al., 2019, AGU Fall Meeting Abstracts, \#SH33A

Simões P. J. A., Hudson H. S., Fletcher L., 2015a, Sol. Phys., 290, 3625

Simões P. J. A., Graham D. R., Fletcher L., 2015b, Sol. Phys., 290, 3573

Simões P. J. A., Reid H. A. S., Milligan R. O., Fletcher L., 2019, ApJ, 870, 114

Sterling A. C., Hudson H. S., Watanabe T., 1997, ApJ, 479, L149

Thomas R. J., Starr R., Crannell C. J., 1985, Sol. Phys., 95, 323

White S. M., Thomas R. J., Schwartz R. A., 2005, Sol. Phys., 227, 231

This paper has been typeset from a $\mathrm{T}_{\mathrm{E}} \mathrm{X} / \mathrm{L} \mathrm{AT} \mathrm{E}$ file prepared by the author. 